\title{
SEISMIC RELIABILITY ASSESSMENT OF FRAME STRUCTURES WITH FLEXIBILITY-BASED STOCHASTIC BEAM-COLUMN ELEMENTS
}

\author{
Georgios Balokas $^{1,2}$ and Michalis Fragiadakis ${ }^{1}$ \\ ${ }^{1}$ Laboratory for Earthquake Engineering, School of Civil Engineering, \\ National Technical University of Athens, Athens, GR-15773, Greece \\ e-mail: Georgios.Balokas@elan-ausy.com, mfrag@mail.ntua.gr \\ ${ }^{2}$ Structure Development Department, ELAN-AUSY GmbH, \\ Harburger Schloßstr. 24, Hamburg, Germany
}

Keywords: Non-Gaussian translation fields, Fiber-approach, Distributed plasticity, Stochastic analysis.

\begin{abstract}
We study the nonlinear static and dynamic analysis of problems with uncertain, spatially variable material properties using specifically tailored flexibility-based fiber elements. For deterministic problems, flexibility, or force-based, fiber elements have been proven able to provide accurate response estimates using a single beam element per structural member. We extent this formulation for the probabilistic assessment of structures whose properties are described by homogeneous non-Gaussian translation stochastic fields. The proposed modeling allows the use of different integration schemes, depending on the correlation length parameter of the stochastic field. This element formulation, contrary to ordinary displacement-based elements, allows to overcome the need for a very dense mesh of beam elements, which depends on the stochastic field and the regions where plastic rotations will occur. The performance of the proposed modeling approach is demonstrated on a steel portal frame. Considerable reduction of the computing effort is achieved in all cases. The paper underlines the importance of realistic uncertainty quantification and is expected to provide valuable guidance for the structural analysis and the design of systems with uncertain properties.
\end{abstract}




\section{INTRODUCTION}

The stochastic approach continuously gains acceptance as a reliable and valuable tool for the calculation of the response variability of structures. The inherent randomness of certain systems in terms of material, geometry and loads prohibits deterministic treatment of the problem. Over the past 20 years the seismic reliability assessment of structures including uncertainties, has received considerable attention. The spatial variability of material and geometrical properties [1] along with the uncertainty of seismic excitation [2] and the coupling of stochasticity and nonlinearity [3], have been thoroughly investigated with various approaches.

Fiber elements have been used on many occasions for the reliability assessment of frame structures. Contrary to the deterministic problem where inelastic deformations are lumped at the beam ends, in stochastic analysis we need distributed plasticity elements so that our calculations consider the variation of system properties along the member [4]. The use of forcebased fiber beam-column elements allows to consistently integrate the spatial variability of inelastic systems with uncertain system properties. For deterministic problems these elements are able to provide accurate response estimates using a single beam element per member. The use of force-based elements for the stochastic assessment of steel frames was first presented in [5] where the seismic capacity of a steel frame subjected to natural ground motion records was examined. In [6] the force-based formulation for the reliability assessment of bridges was used under moving loads, while [7] used force-based fiber elements for the nonlinear static assessment of a simple two-bay frame. Their numerical results are based on a series of measurements of the system properties [8].

\section{INELASTIC FIBER BEAM-COLUMN ELEMENTS}

\subsection{Distributed plasticity elements}

Distributed plasticity beam-column elements allow yielding to occur at any location along the element as opposed to plastic-hinge elements, where inelastic demand is lumped at the beam ends. The two most common formulations of distributed plasticity elements are the displacement-based (DB) and the force-based (FB) approach.

Displacement-based elements, also known as stiffness-based elements, follow the classical finite element theory and use cubic Hermitian shape functions to interpolate the displacement field. These elements require a fine mesh at the regions where inelastic deformations are expected to be high, e.g. the beam ends. On the other hand, force-based elements $[9,10,11]$ use force interpolation functions to overcome the problem of the unknown curvature distribution once yielding occurs. This approach always maintains equilibrium of both forces and deformations and converges to a state that satisfies the constitutive laws within a specified tolerance. As a result, for deterministic problems a single force-based element per member is sufficient for accurately predicting the nonlinear behavior, provided that no element loads are present.

Nonlinear beam-column elements are usually based on the "natural" coordinate system (also known as "basic" or "corotational" system) which is a system that translates and rotates following the motion of the element. The beam elements have three degrees-of-freedom (Figure 1), the axial displacement $u_{1}$ and two rotations $\theta_{1}$ and $\theta_{2}$ which fully describe the inelastic demand and are grouped in $\mathbf{v}=\left[u_{1}, \theta_{1}, \theta_{2}\right]^{\mathrm{T}}$. Following the Euler-Bernoulli beam theory, the strain $\varepsilon_{x}(x, y)$ is obtained as:

$$
\varepsilon_{x}(x, y)=\left[\begin{array}{ll}
1 & -y
\end{array}\right]\left[\begin{array}{c}
\varepsilon_{0}(x) \\
k(x)
\end{array}\right]=\mathbf{a}_{S}(y) \mathbf{d}_{\mathrm{sec}}(x)
$$


where $\mathbf{a}_{\mathrm{s}}(y)$ is the section kinematic matrix and $\mathbf{d}_{\mathrm{sec}}(x)$ is the section deformation vector. The section stiffness matrix is calculated as the derivative of the section forces $\mathbf{D}_{\mathrm{sec}}$ with respect to the section deformations $\mathbf{d}_{\mathrm{sec}}$ :

$$
\mathbf{k}_{\mathrm{sec}}=\frac{\partial \mathbf{D}_{\mathrm{sec}}}{\partial \mathbf{d}_{\mathrm{sec}}}=\frac{\partial \mathbf{D}_{\mathrm{sec}}}{\partial \sigma} \frac{\partial \sigma}{\partial \varepsilon} \frac{\partial \varepsilon}{\partial \mathbf{d}_{\mathrm{sec}}}=\int_{A} \mathbf{a}_{S} \frac{\partial \sigma}{\partial \varepsilon} \mathbf{a}_{S}^{\mathrm{T}} d A=\int_{A} \frac{\partial \sigma}{\partial \varepsilon}\left[\begin{array}{cc}
1 & -y \\
-y & y^{2}
\end{array}\right] d A
$$

where $\partial \sigma / \partial \varepsilon$ is the tangent of the nonlinear uniaxial constitutive law and $y$ is the distance from the neutral axis. Distributed plasticity elements are also known as "fiber" elements, since each section is discretized to a finite number of fibers which are used to numerically calculate the section stiffness $\mathbf{k}_{\mathrm{sec}}$ of Eq. (2). If $N$ is the axial force and $M$ is the bending moment of a crosssection, the section forces are calculated by integrating the stress over the height of the crosssection as follows:

$$
\mathbf{D}_{\text {sec }}=\left[\begin{array}{c}
N \\
M
\end{array}\right]=\int_{A}\left[\begin{array}{c}
1 \\
-y
\end{array}\right] \sigma_{x} d A=\int_{A} \mathbf{a}_{S}^{\mathrm{T}} \sigma_{x} d A
$$

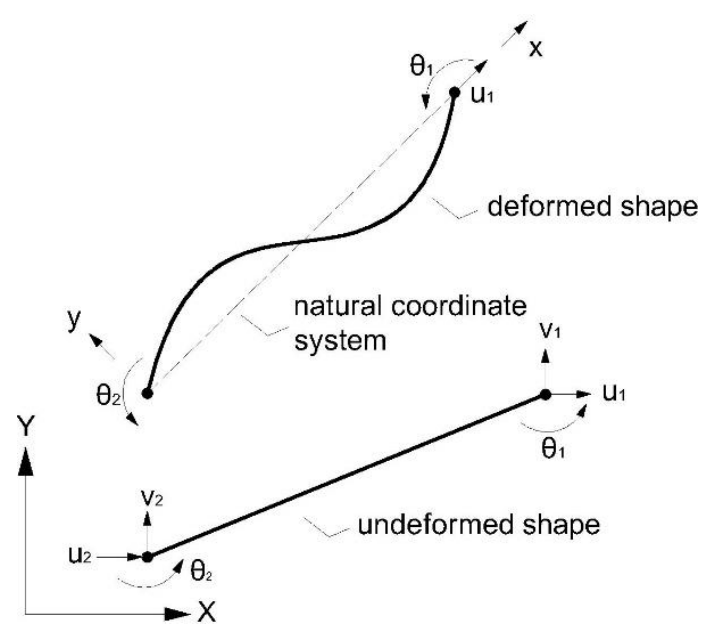

Figure 1: Cartesian and natural coordinates of a plane beam-column element.

\subsection{Displacement-based elements}

The displacement-based (DB) method uses the element interpolation functions and its derivatives in order to calculate section deformations and strains from the nodal displacements. The relationship of section and element deformations is given by:

$$
\mathbf{d}_{\mathrm{sec}}=\mathbf{B}_{N}(x) \mathbf{v}=\frac{1}{L}\left[\begin{array}{ccc}
1 & 0 & 0 \\
0 & 2(3 x / L-2) & 2(3 x / L-1)
\end{array}\right] \mathbf{v}
$$

$\mathbf{B}_{N}(x)$ is the strain-displacement transformation matrix of the element. The element stiffness matrix is calculated in the natural system as:

$$
\mathbf{K}_{\mathrm{N}}=\int_{L} \mathbf{B}_{\mathrm{N}}^{\mathrm{T}} \mathbf{k}_{\mathrm{sec}} \mathbf{B}_{\mathrm{N}} d L
$$

The Cartesian element stiffness is obtained from $\mathbf{K}_{\mathrm{N}}$ with the aid of simple algebraic transformations [12]. 


\subsection{Force-based elements}

The force-based (FB) method uses force interpolation functions, which are always exact since the distribution of bending moments remains linear after element yielding. The force interpolation matrix $\mathbf{b}_{\text {s }}$ relates section forces with the natural forces $\mathbf{S}$, thus:

$$
\mathbf{D}_{\mathrm{sec}}=\mathbf{b}_{\mathrm{S}} \mathbf{S} \Leftrightarrow \mathbf{D}_{\mathrm{sec}}=\left[\begin{array}{ccc}
1 & 0 & 0 \\
0 & x / L-1 & x / L
\end{array}\right] \mathbf{S}
$$

The natural stiffness matrix is calculated as the inverse of the element flexibility matrix as:

$$
\mathbf{K}_{N}^{-1}=\mathbf{F}_{N}=\int_{L} \mathbf{b}_{S}^{T}\left(\mathbf{k}_{\mathrm{sec}}\right)^{-1} \mathbf{b}_{S} d L
$$

For both displacement and force-based elements, the element stiffness matrix is calculated numerically using Gauss integration. For the case of force-based elements, Gauss-Lobatto integration is preferred which is a variation of Gauss integration that considers the beam ends as integration sections. This rule is preferred since the bending moment receives its maximum values at the beam ends.

\section{THE STOCHASTIC FINITE ELEMENT METHOD}

\subsection{Stochastic field discretization}

For nonlinear inelastic problems the discretization of the stochastic field requires special attention. A stochastic (or random) field $H(\mathbf{x}, \omega)$ is a mapping from a random outcome $\omega$ to a function of space (or time) of a random variable $x$. It is usually called "field" when it varies in space and "process" when it varies in time. The statistical properties of stochastic fields (e.g. probability distribution and correlation structure) are either assumed or obtained from experimental measurements. In the stochastic finite element method the choice of the FE mesh size is critical, since this decision affects also the discretization of the stochastic field. In principle, the FE mesh size is controlled by the geometry and the expected gradient of the stress field, which in nonlinear problems is not constant during analysis, while the variation of the stochastic field is a property of the structure. The variation of the stochastic field is usually measured using the correlation length parameter $b$, which is the distance over which significant loss of correlation occurs. Therefore, the FE mesh should be short enough in order to capture the essential features of the random field and avoid loss of information.

\subsection{Simulation of non-Gaussian stochastic fields}

Non-Gaussian stochastic fields are suitable for the description of many practical engineering parameters, such as material properties, geometric characteristics, soil properties, waves, wind loads, etc. In order to simulate a non-Gaussian stochastic field, a transformation of a Gaussian field with known second-order statistics needs to be performed. The spectral representation method [13] is a direct method for the simulation of Gaussian stochastic fields. The method calculates the stochastic field as the sum of cosine functions with random phase angles and amplitudes. It is based on the power spectrum concept, which is a real, non-negative function that describes how the variance of the stochastic field data is distributed over the frequency domain. Spectral density functions include the variance and the correlation scale characteristics of the stochastic field and usually are functions of exponential or square exponential type.

A power spectrum of square exponential type is shown in Figure $2 \mathrm{a}$ for different values of the correlation length $b$, while the influence of the correlation length parameter to the sample 
functions of a Gaussian stochastic field, generated by the spectral representation method, is shown in Figure 2b.

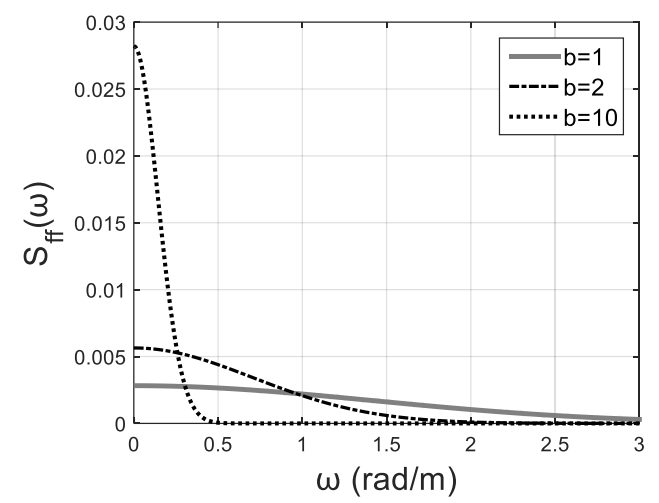

(a)

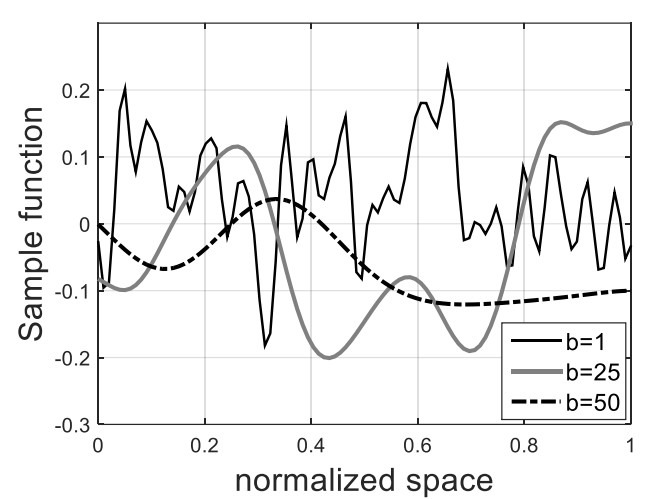

(b)

Figure 2: (a) Sample spectral density function $S_{f f}(\omega)$ of square exponential type with standard deviation $\sigma_{f f}=0.1$ and different correlation length values, (b) sample functions of a Gaussian stochastic field for different values of the correlation length parameter $b$.

A non-Gaussian stochastic field can be obtained as a translation field by properly transforming a Gaussian field. Specifically, a zero-mean homogeneous non-Gaussian stochastic field $f(\mathbf{x})$ with spectral density function $S_{f f}(\omega)$, can be obtained with the aid of a nonlinear monotonic transformation of a zero-mean Gaussian field $H(\mathbf{x})$ as:

$$
f(\mathbf{x})=F^{-1} \Phi[H(\mathbf{x})]
$$

where $F$ is the non-Gaussian marginal cumulative distribution function (CDF) of $f(\mathbf{x})$ and $\Phi$ is the standard Gaussian CDF. The above transformation is a memory-less translation of every space coordinate $x_{i}$. However, the choice of the marginal distribution for the translation field $f(x)$ imposes constraints to its correlation structure [14]. For non-Gaussian translation fields whose autocorrelation function has some inadmissible values, or is not positive-definite, the approximation error should be also taken into consideration [15].

\section{PROPOSED METHODOLOGY}

We propose the use of flexibility-based elements for the seismic probabilistic assessment of nonlinear frame structures with stochastic properties. For the deterministic analysis of inelastic frame structures, flexibility-based elements are able to capture the response using a single element per member. When stochastic problems are considered, the frame properties vary along the length of every member. Most FE types require appropriately modifying the FE mesh depending on the properties of the stochastic field and also on the regions where concentration of inelastic demand is expected, i.e. beam ends, region of concentrated forces etc. However, for stochastic problems the critical locations are not known a priori since the structural properties vary. We show that force-based elements, if combined with a pertinent numerical integration scheme, offer accurate estimates of the response variability, maintaining the advantage of a single element per member. The resulting FE scheme is suitable for full-scale frame structures with affordable computing cost and accuracy.

We use stochastic non-Gaussian fields to simulate the material properties. Constitutive laws typically depend on several parameters. A simple bilinear model depends on two parameters: the elastic modulus $E$ and the yield stress $f_{\mathrm{y}}$, while more parameters may be required for other materials, e.g. reinforced concrete. The material parameters may be denoted as $D_{\mathrm{i}}$ and 
are here assumed uncorrelated. If $H(\mathbf{x})$ is the zero-mean stochastic field, each material property is distributed along each member. If $D_{0, \mathrm{i}}$ is the expected value of each parameter the material property is described as $D_{\mathrm{i}}(\mathbf{x})=D_{0, \mathrm{i}}\left(1+H_{\mathrm{i}}(\mathbf{x})\right)$. The section stiffness $\mathbf{k}_{\mathrm{sec}}$ is calculated with the aid of Eq. (2), where the material properties are a function of $\mathbf{D}=\left[D_{1}, D_{2}, \ldots, D_{\mathrm{N}}\right]^{\mathrm{T}}$. For example for a bilinear steel material $\mathbf{D}=\left[E, f_{\mathrm{y}},\right]^{\mathrm{T}}$ and the section stiffness is obtained with the aid of Eq. (2):

$$
\mathbf{k}_{\mathrm{sec}}=\int_{A} \mathbf{a}_{S}^{\mathrm{T}} \frac{\partial \sigma(\mathbf{D})}{\partial \varepsilon} \mathbf{a}_{S}^{\mathrm{T}} d A
$$

The element stiffness matrix is calculated at the Gauss-Lobatto integration sections as:

$$
\begin{aligned}
& \mathbf{K}_{N}=\int_{L} \mathbf{b}^{\mathrm{T}} \mathbf{k}_{\mathrm{sec}}^{-1} \mathbf{b}^{\mathrm{T}} d L \Leftrightarrow \\
& \mathbf{K}_{N}=w_{1} \mathbf{b}^{\mathrm{T}}\left(x_{1}\right) \mathbf{k}_{\mathrm{sec}}^{-1}\left(x_{1}\right) \mathbf{b}\left(x_{1}\right)+w_{N I P} \mathbf{b}^{\mathrm{T}}\left(x_{N I P}\right) \mathbf{k}_{\mathrm{sec}}^{-1}\left(x_{N I P}\right) \mathbf{b}\left(x_{N I P}\right)+ \\
& +\sum_{i=2}^{N I P-1} w_{i} \mathbf{b}^{\mathrm{T}}\left(x_{i}\right) \mathbf{k}_{\mathrm{sec}}^{-1}\left(x_{i}\right) \mathbf{b}\left(x_{i}\right)
\end{aligned}
$$

where NIP is the number of integration points, $w_{i}$ and $x_{i}$ are the weights and the location of Gauss-Lobatto integration sections. Note that $x_{1}$ and $x_{N I P}$ refer always to the two ends of the beam element. A similar integration is also adopted for the element internal forces. The above methodology is implemented with the aid of OpenSees [16]. OpenSees is an open-source structural analysis software that allows the user to define any numerical integration scheme. The pre and post processing of our results are performed with the aid of customized in-house software.

We first generate zero-mean Gaussian stochastic fields using the spectral representation method. These fields have a spectral density function of square exponential type:

$$
S_{H H}(\omega)=\frac{\sigma^{2} b}{2 \sqrt{\pi}} \exp \left(-\frac{b^{2} \omega^{2}}{4}\right)
$$

where $\sigma$ is the standard deviation of the stochastic field and $b$ is the correlation length parameter. The lognormal fields are subsequently obtained using Eq. (8). The spectral density function is expected to be slightly different from $S_{H H}(\omega)$, since the translation to the lognormal $\mathrm{CDF}$ adds a small approximation error.

\section{STEEL PORTAL FRAME EXAMPLE}

We consider the steel portal frame of Figure 3 as a case study. All the members of the frame have HEB 200 wide flange cross-sections and are modeled with a single force-based beam-column element. A distributed load $\mathrm{q}=40 \mathrm{kN} / \mathrm{m}$ is applied at the bay and remains constant throughout the loading history. The uncertain material properties are the Young's modulus $E$ and the yield stress $f_{y}$, both assumed to vary stochastically along each member. The material properties are described by the following expressions:

$$
\begin{aligned}
& E(x)=E_{0}\left[1+H_{1}(x)\right] \\
& f_{y}(x)=f_{y 0}\left[1+H_{2}(x)\right]
\end{aligned}
$$

where $H_{1}(\mathrm{x}), H_{2}(\mathrm{x})$ are two zero-mean 1D-1V homogeneous lognormal stochastic fields with COV equal to $10 \%$ as indicated by the Joint Committee for Structural Safety [17]. Different values of the correlation length parameter $b$ are considered in order to investigate the sensitiv- 
ity of the response to the correlation scale of the stochastic fields. In order to validate the proposed method, we consider as reference solution FE models that use a very dense mesh of 50 displacement-based fiber elements per member. Such a dense mesh is expected to accurately describe randomness for any correlation length.

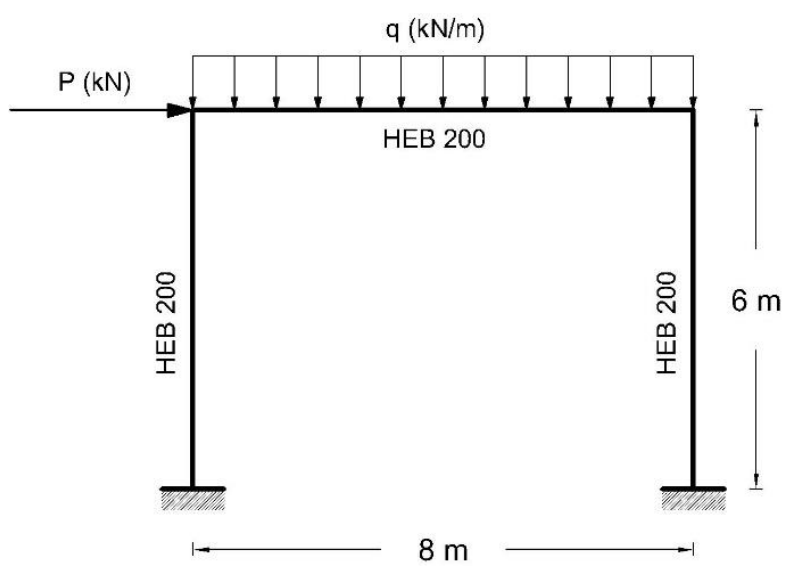

Figure 3: One-storey steel frame.

\subsection{Nonlinear static analysis}

The response variability of the steel frame is calculated through nonlinear static analysis for a sample size of 500 crude Monte Carlo simulations. For every simulation we obtain the capacity curve in terms of roof drift versus applied load. The mean capacity curve of the frame is shown in Figure 4a, together with the mean plus and minus one standard deviation curves. The vertical dashed line is used in order to separate the pre-yielding from the postyielding phase of loading. Figure 4a clearly shows that the effect of $E$ is rather small (preyielding phase), while considerable variability is observed after yielding where $f_{y}$ comes into play.

According to Figure $4 \mathrm{~b}$ the calculation of the mean capacity is not sensitive to the number of integration sections even for a small correlation length $(b=0.1)$. On the other hand, Figure 5 examines the necessary number of integration points in order to capture the response variability defined as the COV (standard deviation divided by the mean value) conditional on the roof drift. As a reference solution we use the red solid curve obtained with the aid of a very dense mesh of 50 displacement-based elements per member. For four correlation length values, we compare the COV estimates using force-based elements of 5, 10, 15 and 20 integration sections. All four plots of Figure 5 show that increasing the number of integration points offers a better description of the random fields, since the COV values converge to the exact solution. The convergence is faster as the correlation length $b$ becomes larger (e.g. compare Figure 5a and Figure 5d). The vertical dashed line (defined in Figure 4) provides the threshold between linear elastic and inelastic response. In the elastic regions the COV is practically constant and very low (2-6\%). When the frame starts to yield, the COV increases almost monotonically as the roof drift also increases. 


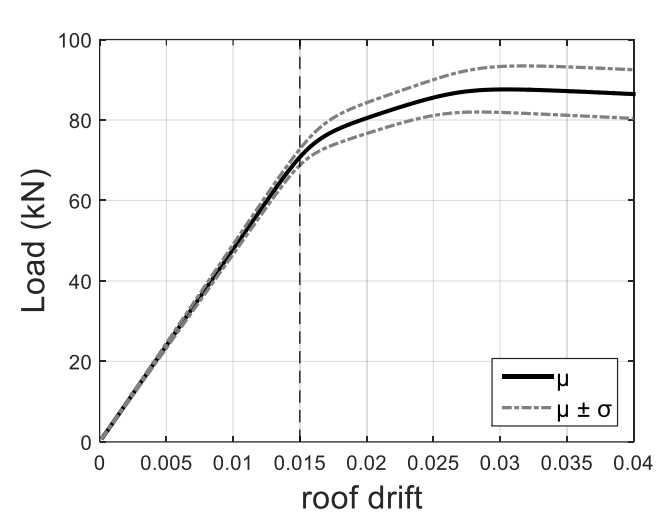

(a)

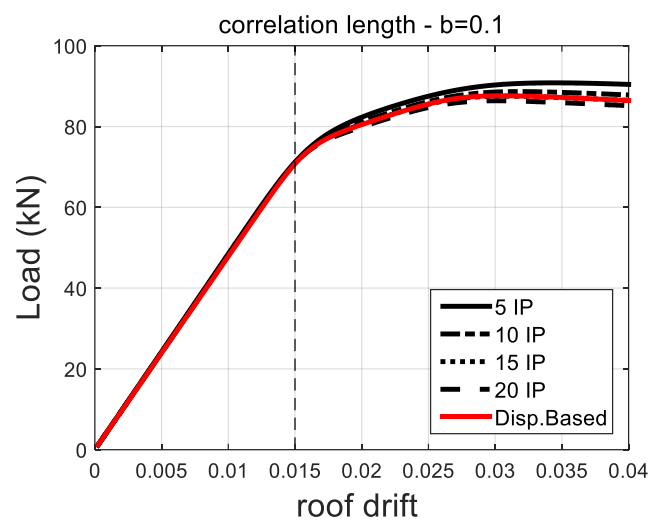

(b)

Figure 4: (a) Mean and mean \pm one sigma capacity curves of the portal frame, (b) mean capacity curves for ranging number of integration points.

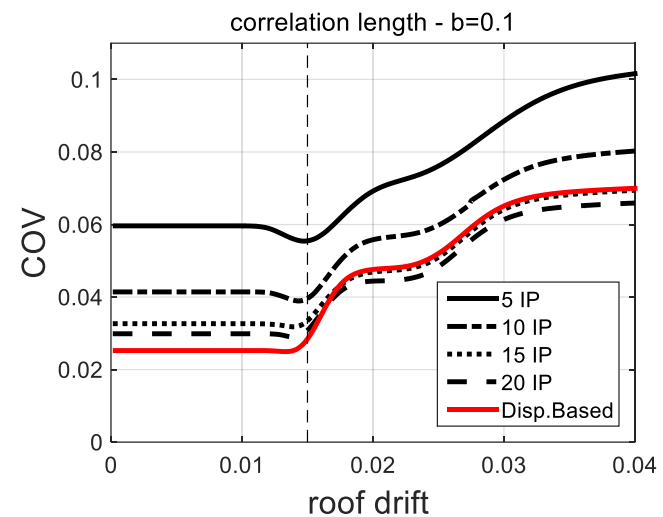

(a)

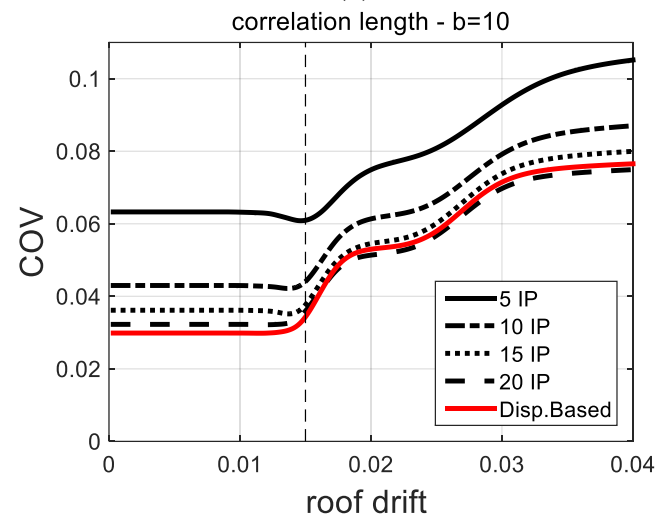

(c)

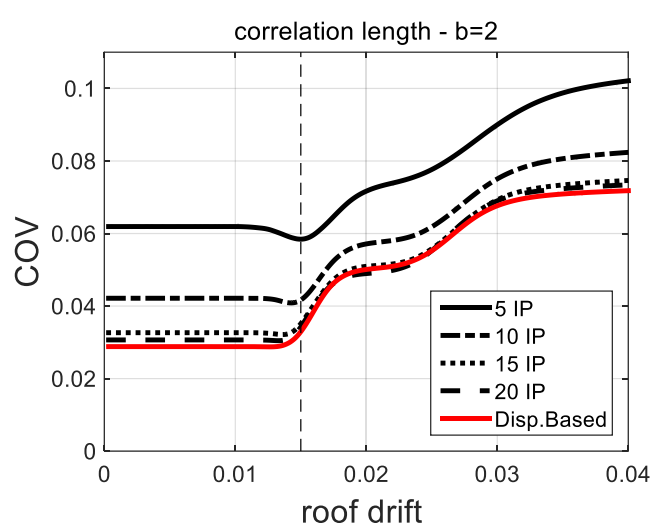

(b)

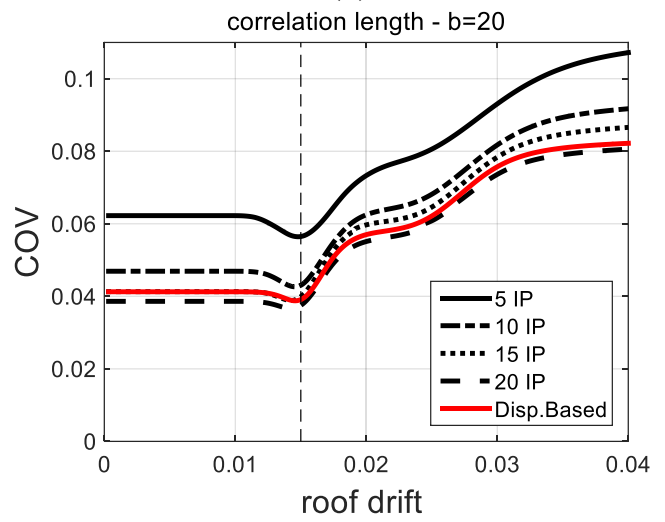

(d)

Figure 5: Sensitivity of response variability (COV) to the number of integration points for different correlation lengths.

\subsection{Nonlinear response history analysis}

The seismic performance of the steel portal frame is also studied using nonlinear response history analysis. Seismic demand is measured with the aid of the maximum roof drift ratio $\left(\theta_{\max }\right)$. A lumped mass matrix is formed in agreement to the distributed load $q$ (Figure 3 ). The fundamental mode of the frame was found equal to $T_{1}=1.24 \mathrm{sec}$ when the mean value of the Young's modulus $E$ is used, while the damping matrix was obtained assuming 5\%-Rayleigh 
damping on the first and the second mode. All response history analyses were performed using a single force-based beam-column element per member with 20 Gauss-Lobatto integration sections. Again we assume that the correct solution is that of a very dense mesh of displacement-based elements.

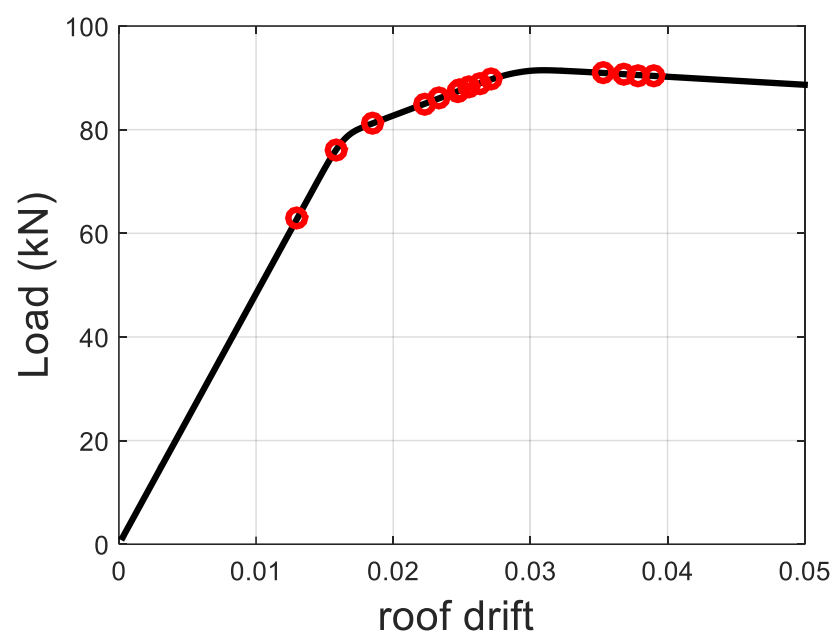

Figure 6: Roof drift demand of the natural ground motion records (red circles) plotted on the capacity curve of the frame (solid line).

All response history analyses are performed with fifteen ground motion records. The records cover a broad spectrum of seismic intensities in order to evaluate the structural behavior at different levels of seismic demand. All records are scaled with a uniform scaling factor equal to 2, to guarantee that some of them will yield the frame. Figure 6 shows the maximum drifts demand for every record plotted against the capacity curve of the frame, assuming the mean value of every random parameter. Significant record-to-record variability is expected due to the different frequency content and duration of the ground motions.

The accuracy of the proposed method is examined considering the first three statistical moments. Figure 7 shows the mean storey drift $\left(\theta_{\max }\right)$ for four values of the correlation length. The proposed force-based modeling provides results that are practically identical to the "correct" solution. Note that in Figure 7, the records are sorted from left to right according to the maximum expected drift demand of Figure 6.

Figure 8 shows the effect of stochastic material properties on the COV of $\theta_{\max }$ demand. For all $b$ values considered, the accuracy of the force-based formulation is again very close to the correct solution. The errors observed are small proving that the discretization with 20 sections is sufficient. Furthermore, records that do not cause large inelastic displacement demand (records on the left) have smaller COV values than records that caused inelastic damage on the building (records towards the right). Still, the COV values are very sensitive to the record characteristics while, for most ground motions, the COV of the response is lower than the variance of the stochastic input parameters. The effect of the correlation length is small compared to the significance of the ground motion properties, while large correlation lengths tend to increase the COV. 

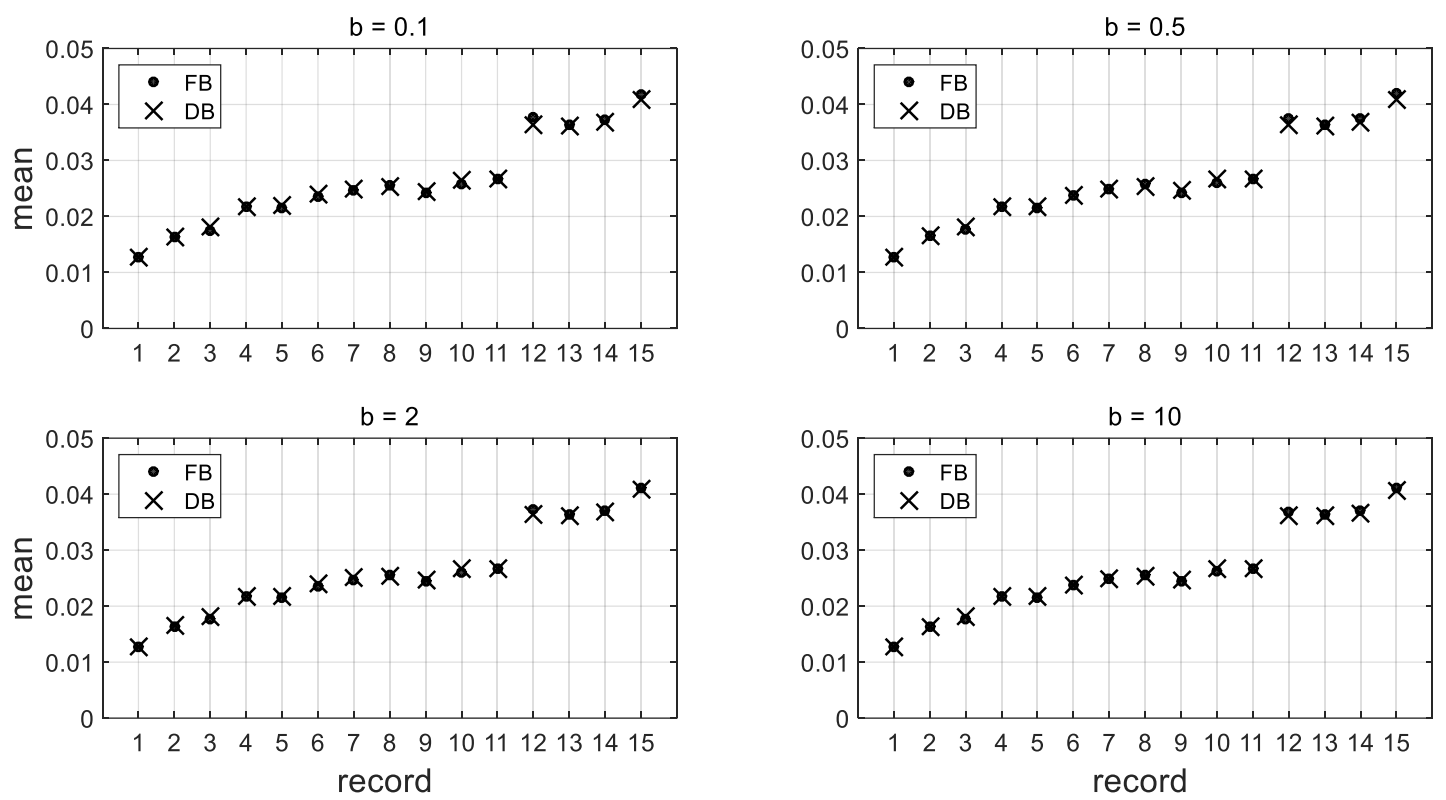

Figure 7: Mean drift demand for correlation values equal to $b=0.1,0.5,2$ and 10 .
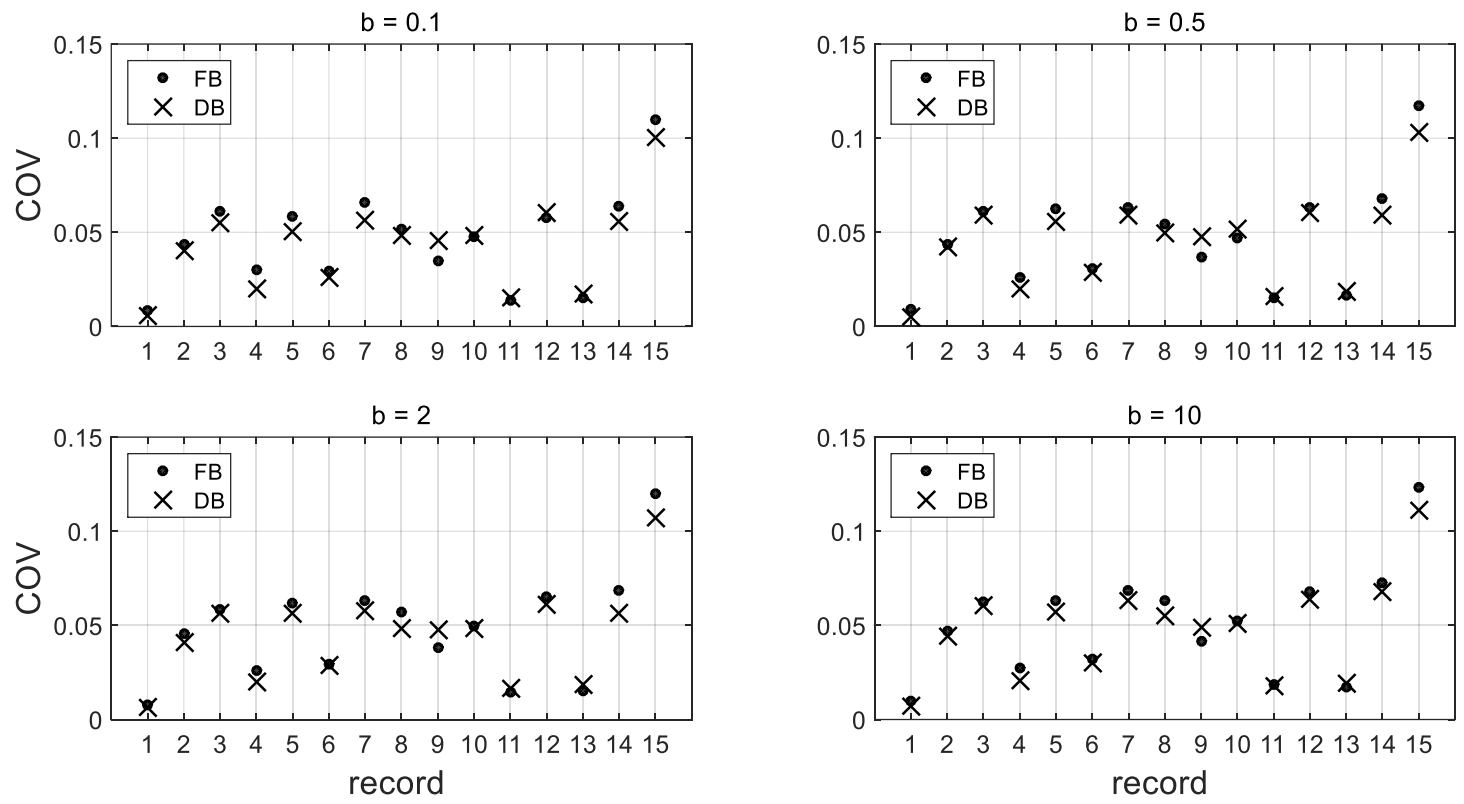

Figure 8: COV of drift demand for correlation values equal to $b=0.1,0.5,2$ and 10 .

Figure 9 shows the skewness (third statistical moment). The skewness provides a measure of the asymmetry of the sample's cumulative distribution function. Contrary to the COV, the skewness is quite sensitive to the correlation length and varies with the record properties. For many records the skewness differs considerably for different $b$ values and doesn't follow the properties of the lognormal material/input properties. Furthermore, samples of opposite skewness for the same natural record, e.g. records 11 and 15, were found. In all cases, the proposed modeling gave excellent estimates of the skewness. 

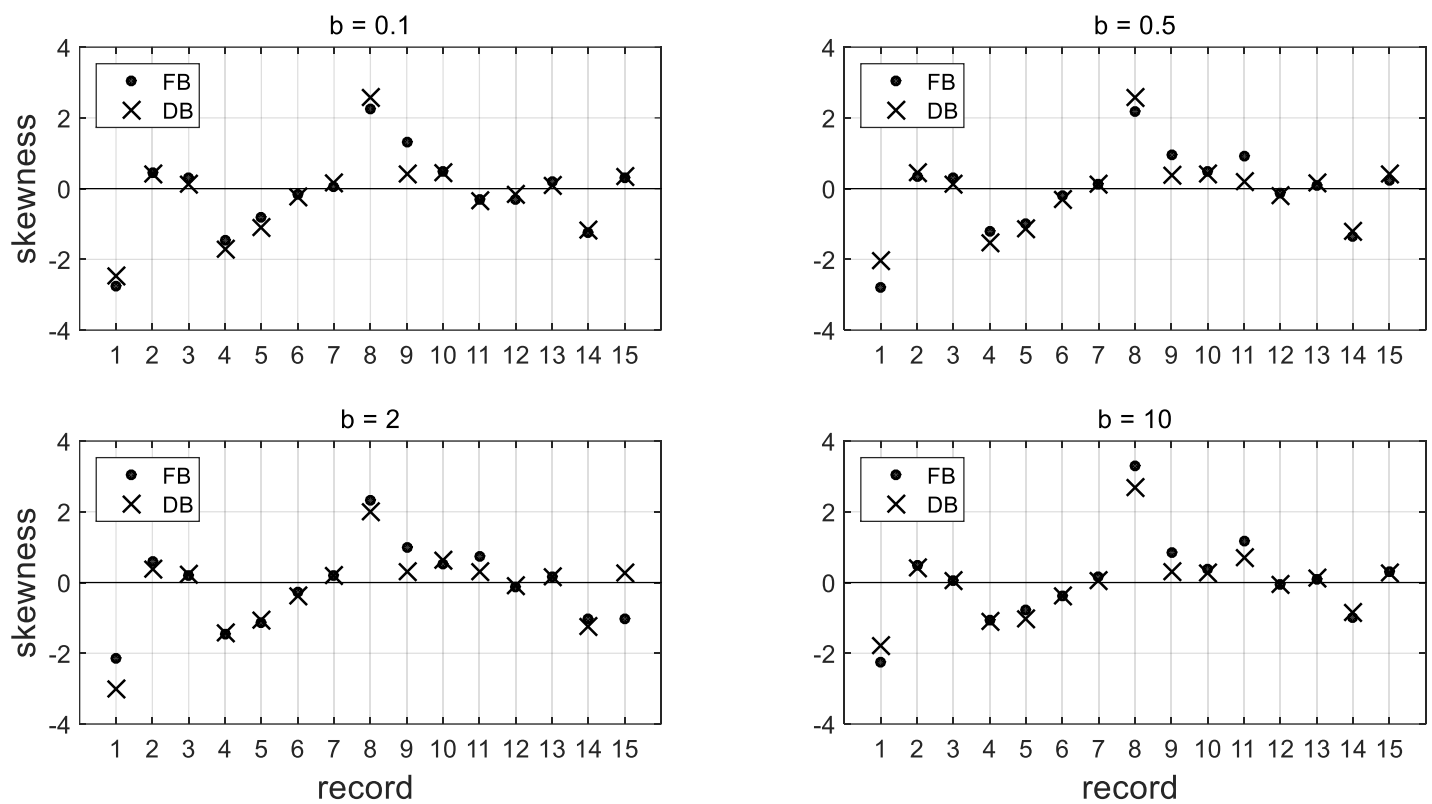

Figure 9: Skewness of drift demand for correlation values equal to $b=0.1,0.5,2$ and 10.

Apart from accuracy, the proposed method also reduces considerably the computing cost. Figure 10 compares the computational cost of the proposed element to that of the displacement-based formulation for both static and dynamic analysis. An Intel Core 2 Duo processor required more than double time to run 500 Monte Carlo simulations for the nonlinear static case. For the response history analysis of a single record, the proposed methodology required approximately $10 \%$ of the time of the displacement-based element for the same number of simulations. Although the computational effort of the displacement-based element can be reduced using a more coarse mesh, Figure 10 provides a clear indication of the exceptional performance of the force-based element which can be therefore adopted for the simulation of real-scale problems without having to modify the mesh of fiber elements.

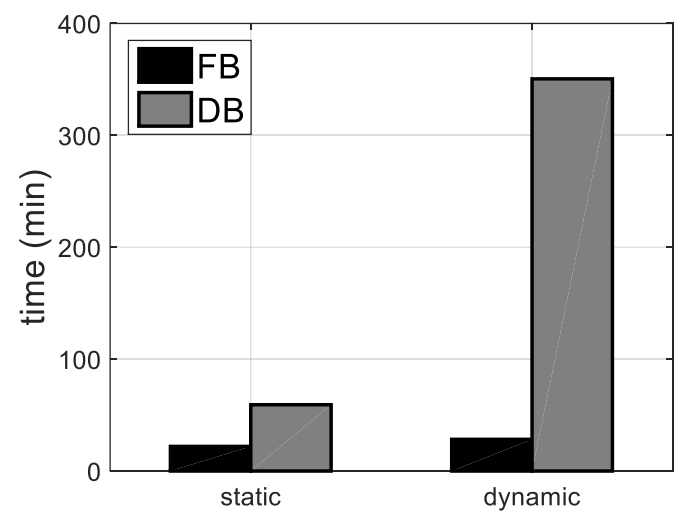

Figure 10: Computing cost of 500 Monte Carlo simulations of the portal frame for the force-based and the displacement-based formulation.

\section{CONCLUSIONS}

A novel modeling approach for the probabilistic seismic assessment of frame structures with stochastic system properties is proposed. The proposed method extends the use of flexi- 
bility-based fiber elements to the stochastic finite element method, offering the possibility of applications to real-scale problems due to the remarkable computational performance and stability of these elements. The performance of the proposed modeling is demonstrated on a onestorey steel portal frame. The study provides a valuable guidance for the analysis and the design of structures with non-Gaussian system properties and its main conclusions are here summarized:

- The choice of the number of integration points is essential. The integration should be able to capture the spectral characteristics of the input stochastic field.

- The proposed modeling offers remarkable accuracy and reduced computing numerical effort, even for very small values of the correlation length parameter (highly uncorrelated stochastic fields).

- The computational performance was exceptional, especially for the case of response history analysis where the proposed modeling reduced the required computing effort by $90 \%$. This allows the implementation of the method to real-scale problems.

\section{REFERENCES}

[1] S. Chakraborty, S.S. Dey, Stochastic finite element method for spatial distribution of material properties and external loading. Computers and Structures, 55, 41-45, 1995.

[2] I.A. Kougioumtzoglou, P.D. Spanos, An approximate approach for nonlinear system response determination under evolutionary stochastic excitation. Current Science, 97(8), 1203-1211, 2009.

[3] J-B. Chen, J. Li, Stochastic seismic response analysis of structures exhibiting high nonlinearity. Computers and Structures, 88, 395-412, 2010.

[4] T-H. Lee, M. Mosalam, Probabilistic fiber element modeling of reinforced concrete structures. Computers and Structures, 82, 2285-2299, 2004.

[5] G. Stefanou, M. Fragiadakis, Nonlinear dynamic analysis of frames with stochastic nonGaussian material properties. Engineering Structures, 31, 1841-1850, 2009.

[6] O.M. Hamutcuoglu, M.H. Scott, Finite element reliability analysis of bridge girders considering moment-shear interaction. Structural Safety, 31, 356-362, 2009.

[7] D. Feng, J. Li, Stochastic nonlinear behaviour of reinforced concrete frames. II: Numerical Simulation. Journal of Structural Engineering, DOI: 10.1061/(ASCE)ST.1943541X.0001443, 2015.

[8] J. Li, D. Feng, X. Gao, Y. Zhang, Stochastic nonlinear behaviour of reinforced concrete frames. I: Experimental investigation. Journal of Structural Engineering, DOI: 10.1061/(ASCE)ST.1943-541X.0001442, 2015.

[9] C. Zeris, S.A. Mahin, Analysis of reinforced concrete beam-columns under uniaxial excitation. Journal of Structural Engineering, 114(4), 804-820, 1988.

[10] E. Spacone, V. Ciampi, F. Filippou, Mixed formulation of nonlinear beam element. Computers and Structures, 58(1), 71-83, 1996.

[11] A. Neuenhofer, F. Filippou, Geometrically nonlinear flexibility-based frame finite element. Journal of Structural Engineering, 124(6), 704-711, 1998. 
[12] M. Fragiadakis, Nonlinear analysis of steel and RC frame structures. Laboratory for Earthquake Engineering, Class notes, Athens, Greece, 2016.

[13] M. Shinozuka, G. Deodatis, Simulation of stochastic processes by spectral representation. Applied Mechanics Reviews, 44(4), 191-203, 1991.

[14] M. Grigoriu, Simulation of stationary non-Gaussian translation processes. Journal of Engineering Mechanics, 124(2), 121-126, 1998.

[15] P. Bocchini, G. Deodatis, Critical review and latest developments of a class of simulation algorithms for strongly non-Gaussian random fields. Probabilistic Engineering Mechanics, 23, 393-407, 2008.

[16] F. McKenna, G.L. Fenves, The OpenSees command language manual version 1.2. Pacific Earthquake Engineering Research Centre, University of California, Berkeley, 2001.

[17] Joint Committee for Structural Safety. Probabilistic Model Code. Part 3: Resistance Models, 2001. 